\title{
Update on myocardial perfusion imaging: role of regadenoson
}

\author{
Robert C Hendel \\ Midwest Heart Specialists, Central \\ DuPage Hospital, Winfield, Illinois, USA
}

\begin{abstract}
Pharmacologic stress myocardial perfusion imaging (MPI) is a noninvasive method for the evaluation of coronary artery disease in patients unable to exercise adequately. Commonly used pharmacologic stress agents (adenosine, dipyridamole, and dobutamine) have a high incidence of bothersome and potentially serious side-effects, several contraindications to testing, and require continuous infusion with weight-adjusted doses. Newer agents are, therefore, needed. Selective activation of $\mathrm{A}_{2 \mathrm{~A}}$ adenosine receptors on the coronary vasculature should provide coronary vasodilation for MPI with fewer or less severe side-effects associated with nonspecific agonists, such as adenosine and dipyridamole. Regadenoson is the first selective $\mathrm{A}_{2 \mathrm{~A}}$ receptor agonist to be approved as a pharmacologic stress agent for use with radionuclide MPI. Regadenoson is administered at a standard, fixed dose of $400 \mu \mathrm{g}$ from a prefilled syringe or single-use vial by intravenous injection over 10 seconds. In clinical trials, regadenoson provided good quality images yielding accurate diagnostic information, and was safe and well tolerated. No infusion pump is required and the stress protocol is brief. Further studies are now required to evaluate regadenoson as a stress agent in combination with low-level exercise, with prior caffeine intake, and in patients with asthma or chronic obstructive pulmonary disease.
\end{abstract}

Keywords: myocardial perfusion imaging, pharmacologic stress, $\mathrm{A}_{2 \mathrm{~A}}$ receptor agonist, regadenoson, ischemic heart disease, SPECT, diagnosis

\section{Introduction}

Gated single photon emission computed tomography myocardial perfusion imaging (SPECT MPI) is a validated, noninvasive technique for evaluating subjects with known or suspected coronary artery disease (CAD). ${ }^{1,2}$ Stress radionuclide MPI utilizes an intravenously-administered radiopharmaceutical to visualize relative coronary blood flow under conditions of increased heart rate ( $\geq 85 \%$ of the subject's age-predicted maximum heart rate and five metabolic equivalents). ${ }^{2,3}$ Differences in radionuclide activity between tissues supplied by normal and stenotic arteries during stress (resulting from regional heterogeneity of myocardial blood flow) allows identification of perfusion deficits when stress images are compared with those obtained at rest. Information on perfusion defect location, severity, and extent guides diagnostic, prognostic (ie, risk assessment/stratification), and management decisions. ${ }^{1,2}$ In addition, serial stress MPI can be used to monitor disease progression, assess follow-up risk, detect postrevascularization restenosis, and evaluate the efficacy of medical therapy. ${ }^{2}$ Stress MPI may also be performed with cardiac resonance imaging and contrast echocardiography, although this article deals exclusively with radionuclide imaging, as there is no experience with regadenason in conjunction with these other imaging modalities.

The level of increase in coronary blood flow required to acquire good quality scintigraphic images during stress MPI is best achieved through exercise. ${ }^{2,3}$ However, not all subjects are suitable candidates for exercise testing, including those with a large abdominal aortic aneurysm, left bundle branch block, or a pacemaker. 
In addition, age, neurologic and/or orthopedic limitations, co-morbidities (eg, peripheral vascular disease or chronic obstructive pulmonary disease [COPD]), poor fitness level/ lack of motivation, or concomitant medications prevents many patients from attaining an adequate workload through exercise. ${ }^{1,2}$ Pharmacologic stress testing may be considered for these individuals. Contraindications to pharmacologic stress are hypotension, acute coronary syndrome within 24 hours, critical aortic stenosis, severe left main stenosis, severe left ventricular outflow obstruction, decompensated heart failure, or hypersensitivity to the stress agent.

\section{The need for newer pharmacologic stress agents}

The use of cardiac imaging, including stress MPI, has increased dramatically over recent years due to technical improvements and increased clinical application. ${ }^{4}$ Over 7.5 million stress MPI procedures were conducted in the United States in 2007, almost half of which were pharmacologic stress studies (44\%; 3.3 million). Although appropriateness criteria have been published to minimize the number of inappropriate tests conducted, ${ }^{5}$ a further rise in stress testing is expected over forthcoming years, driven by an aging population and an increase in cardiovascular morbidity and mortality associated with obesity, diabetes, and other risk factors. Experience with pharmacologic stress MPI to date has been largely with adenosine and dipyridamole (coronary vasodilators) and dobutamine (an inotropic agent). However, none of these agents provide an ideal method of medicationinduced stress.

Adenosine and dipyridamole both ultimately induce coronary vasodilation by nonselectively activating $\mathrm{A}_{2 \mathrm{~A}}$ adenosine receptors on myocardial vascular smooth muscle cells, although each moiety dose so either in a direct (adenosine) or indirect (dipyridamole) fashion. Both these vasodilators are associated with a high incidence of side-effects (notably, chest pain, flushing, headache, and dyspnea) and carry a risk of bronchoconstriction or sinoatrial or atrioventricular block. ${ }^{2,6,7}$ Persistent or severe side-effects are reversible with aminophylline. Contraindications to adenosine and dipyridamole include bronchoconstrictive or bronchospastic lung disease (eg, asthma or COPD), second or third degree atrioventricular block, allergy to aminophylline, ingestion of caffeine (or other methylxanthine) within 12 hours, and use of dipyridamole within 24 hours (for adenosine). ${ }^{2,8,9}$ Patients with these contraindications tend to undergo pharmacologic stress testing with dobutamine (plus adjunctive atropine, as necessary), even though it is not approved by the
FDA for this indication. Important common side-effects of dobutamine include chest pain, and arrhythmias. ${ }^{10,11}$ Severe side-effects can be treated by intravenous administration of a short-acting $\beta$-blocker. ${ }^{2}$ Uncontrolled hypertension, uncontrolled atrial fibrillation/flutter, serious ventricular ectopic activity, large aortic aneurysm, and $\beta$-blocker use within 24 hours are contraindications to dobutamine. ${ }^{2,11}$ There are also logistic challenges associated with administering adenosine, dipyridamole, and dobutamine as they involve controlled continuous infusions at weight-adjusted doses; hence, they require specialized intravenous tubing and infusion pumps. ${ }^{8,9,12}$

The increasing demand for stress MPI procedures combined with the limitations of more established pharmacologic stress agents highlight the need for newer drugs. Such agents should have more acceptable tolerability profiles, with a lower incidence of serious and bothersome adverse events; fewer limiting contraindications, and simplified protocols to improve laboratory efficiency.

\section{$A_{2 A}$ adenosine receptor agonists}

Adenosine-mediated myocardial vasodilation is mediated through $\mathrm{A}_{2 \mathrm{~A}}$ adenosine receptors on the coronary vasculature. ${ }^{13}$ The high incidence of side-effects observed with adenosine and dipyridamole during stress MPI can be attributed to the nonselective activation of other adenosine receptor subtypes $\left(\mathrm{A}_{1}, \mathrm{~A}_{2 \mathrm{~B}}\right.$, and $\left.\mathrm{A}_{3}\right){ }^{2,14-17}$ For example, $\mathrm{A}_{1}$ receptor stimulation is associated with atrioventricular conduction abnormalities and chest pain, ${ }^{18} \mathrm{~A}_{2 \mathrm{~B}}$ receptor stimulation is associated with peripheral vasodilation and bronchoconstriction, ${ }^{19}$ and $\mathrm{A}_{3}$ receptor stimulation is associated with bronchospasm and atypical chest pain. ${ }^{20}$ Thus, selective activation of $\mathrm{A}_{2 \mathrm{~A}}$ receptors can be expected to provide adequate coronary vasodilation for MPI with fewer or less severe side-effects. ${ }^{21-23}$

Characterization of the $\mathrm{A}_{2 \mathrm{~A}}$ receptor subtype has facilitated the development of $\mathrm{A}_{2 \mathrm{~A}}$ receptor agonists as pharmacologic stress agents for use in radionuclide MPI. These include regadenoson (Lexiscan ${ }^{\mathrm{TM}}$; Astellas Pharma US, Inc., Deerfield, Illinois), the first $\mathrm{A}_{2 \mathrm{~A}}$ receptor agonist to be approved by the FDA for this indication; ${ }^{24}$ binodenoson (CorVue ${ }^{\mathrm{TM}}$; King Pharmaceuticals, Inc., Bristol, Tennessee), ${ }^{.25-32}$ and apadenoson (Stedivaze ${ }^{\mathrm{TM}}$; PGxHealth/ Clinical Data, Inc., Newton, Massachusetts), ${ }^{33}$ which is due to enter Phase III trials in 2009. Compared with adenosine, these agents appear to provide equivalent diagnostic information, produce less hypotension (but greater tachycardia), have no significant effects on atrioventricular node conduction, are 
associated with generally fewer side-effects, are administered by bolus injection in some cases (regadenoson and binodenoson), and offer the potential for use in patients with bronchospastic disease.

The objective of this review is to discuss the methodology, safety, feasibility and clinical applications of regadenoson stress MPI in the evaluation of CAD.

\section{Regadenoson}

\section{Pharmacologic and early clinical data}

Regadenoson (adenosine, 2-[4-[(methylamino)carbonyl]$1 H$-pyrazol-1-yl]-, monohydrate) is a low-affinity human $\mathrm{A}_{2 \mathrm{~A}}$ receptor agonist $\left(K_{i}=1.269 \mu \mathrm{M}\right.$; where $K_{i}$ is the ratio between the rate of drug dissociation from and the rate of drug binding to the receptor). ${ }^{24,34,35}$ Because of the large receptor reserve (spare receptors) for $\mathrm{A}_{2 \mathrm{~A}}$ receptor-mediated coronary vasodilation, a low-affinity agonist such as regadenoson need only occupy a relatively small fraction of target sites to produce a rapid and near maximal vasodilatory response. ${ }^{35}$ Regadenoson has at least 10 -fold lower affinity for $\mathrm{A}_{1}$ receptors $\left(K_{i}>16.46 \mu \mathrm{M}\right)$ than $\mathrm{A}_{2 \mathrm{~A}}$ receptors, and very weak affinity for $A_{2 B}$ and $A_{3}$ receptors (only $22 \%$ of a competitive ligand in in vitro studies was displaced in each case). ${ }^{34,35}$

The regadenoson plasma concentration-time profile in healthy volunteers was tri-exponential and best characterized by a 3-compartment model. ${ }^{36-38}$ Maximum plasma regadenoson concentrations were achieved within 1-3 minutes of the intravenous injection, which paralleled the onset of the hyperemic response. Concentrations then declined with fast distribution phases, followed by a longer terminal elimination phase (terminal elimination half-life $\approx 2$ hours). ${ }^{36,37}$ Urinary excretion was estimated to account for $58 \%$ of the total clearance. Regadenoson clearance, terminal half-life, and volume of distribution showed no dose dependence within the range of $0.1-30 \mu \mathrm{g} / \mathrm{kg}$ in healthy subjects. ${ }^{37}$

No correlation was observed between regadenoson clearance and the covariates of body weight, body mass index, age, or race. ${ }^{24,37,38}$ Patients with renal impairment had diminished regadenoson clearance, which resulted in higher elimination half-life and area under the curve values compared with subjects with normal renal function. ${ }^{38}$ However, the degree of renal dysfunction was not significantly correlated with maximum regadenoson plasma concentration, volume of distribution, or the number or severity of adverse events. These Phase I pharmacokinetic studies indicate that dose adjustment according to body weight or renal function is not necessary. ${ }^{37,38}$
The pharmacodynamic effects of regadenoson on myocardial blood flow were assessed in an open-label, doseranging study in patients undergoing clinically indicated coronary catheterization for the evaluation or treatment of suspected CAD $(n=34) .{ }^{36}$ Single intravenous bolus doses of regadenoson $(10,30,100,300,400$, or $500 \mu \mathrm{g})$ were infused over 10 seconds, and the effect on coronary blood flow velocity was measured by pulsed-wave ultrasonography using an intracoronary Doppler-tipped guidewire. Near peak coronary blood flow velocity ( $\geq 85 \%$ of maximum) was achieved in a mean of 33 seconds (range, 20-40 seconds) and was not dose dependent. The hyperemic response to regadenoson doses of 400 and $500 \mu \mathrm{g}$ were similar in magnitude and duration (Figure 1); mean peak coronary blood flow velocity was maintained at $\geq 2.5$ times baseline for 2.3 and 2.4 minutes, respectively.

\section{Regadenoson SPECT MPI: initial clinical experience}

The level and time course of increased coronary blood flow observed with regadenoson 400 and $500 \mu \mathrm{g}$ in the study by Lieu et $\mathrm{al}^{36}$ were considered adequate to allow maximal radiotracer extraction by the myocardium during MPI procedures. These two doses were, therefore, selected for use in a pilot imaging study to evaluate the clinical utility of regadenoson as a pharmacologic stress agent in conjunction with SPECT MPI. ${ }^{39}$

This multicenter, open-label, Phase II study involved patients with documented reversible ischemic defects on a 6-minute adenosine stress SPECT MPI study 2-46 days previously. ${ }^{39}$ Patients received a single peripheral intravenous bolus of regadenoson $400 \mu \mathrm{g}(\mathrm{n}=18)$ or $500 \mu \mathrm{g}(\mathrm{n}=17)$ over 10 seconds, followed by a saline flush. The radionuclide (Tc-99m sestamibi or Tc-99m tetrofosmin) was infused 10-20 seconds after the saline flush. Three experts evaluated the perfusion patterns (independently and by consensus) on blinded regadenoson and adenosine SPECT images. In the consensus assessment, $100 \%$ of adenosine scans were considered to show myocardial ischemia compared with $89 \%$ $(16 / 18)$ and $82 \%(14 / 17)$ of scans achieved using regadenoson 400 or $500 \mu \mathrm{g}$, respectively. Agreement between adenosine and regadenoson images for the presence of reversible hypoperfusion (based on the majority of the three independent ratings) was $89 \%$ with regadenoson $400 \mu \mathrm{g}$ and $76 \%$ with regadenoson $500 \mu \mathrm{g}$. There were also no significant differences between adenosine and regadenoson images in visual or quantitative (computerized) assessments of defect severity using a 17-segment model. 


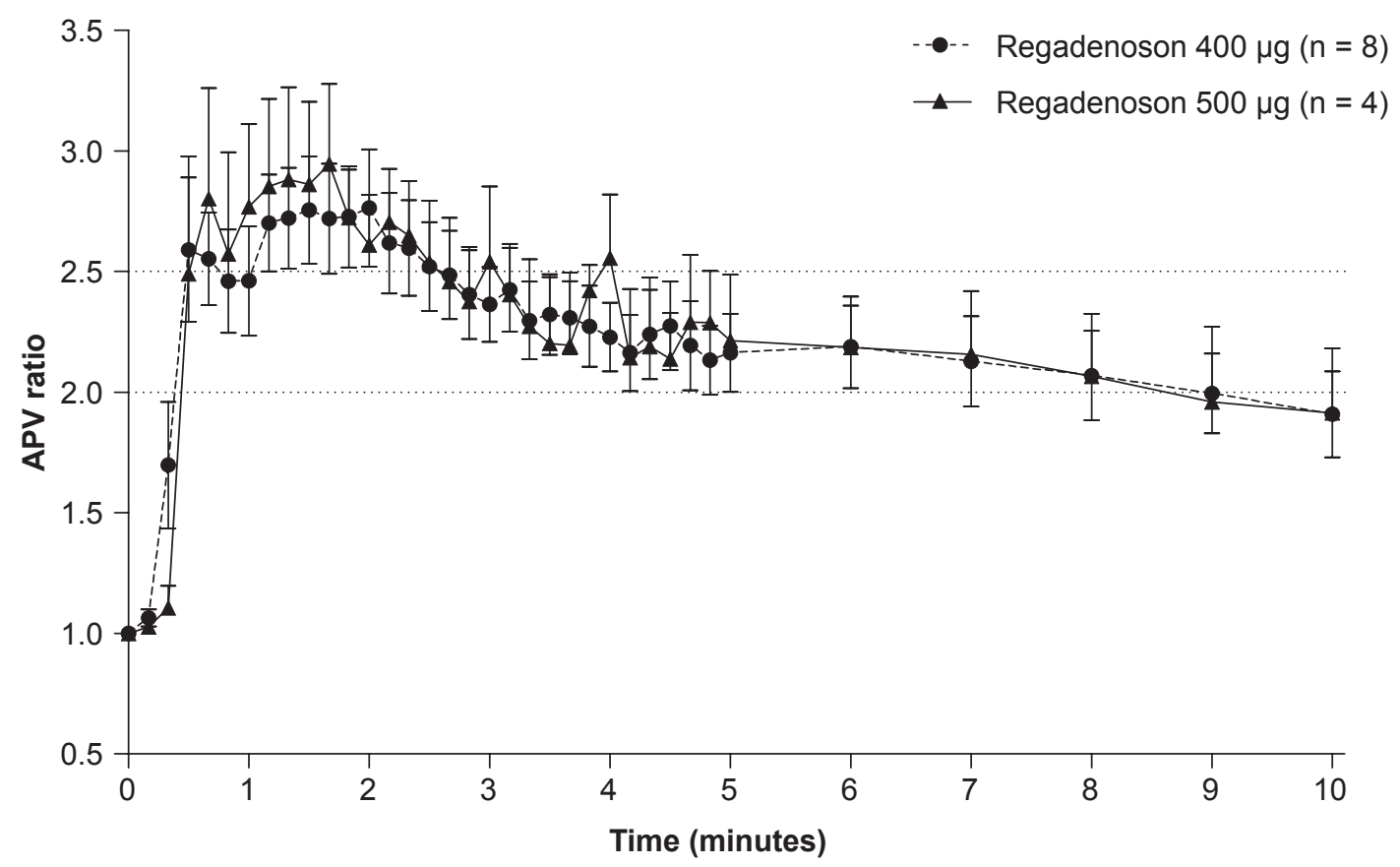

Figure I Time dependence of the effects of regadenoson 400 and $500 \mu \mathrm{g}$ (intravenous bolus) on the ratio of peak-to-baseline average peak blood flow velocity (APV). ${ }^{36} \mathrm{Data}$ are mean \pm SE. Reproduced with permission from Lieu HD, Shryock JC, von Mering GO, et al. Regadenoson, a selective $A_{2 A}$ adenosine receptor agonist, causes dose-dependent increases in coronary blood flow velocity in humans.J Nucl Cardiol. 2007; 14:5 I4-520. ${ }^{36}$ Copyright @ 2007 American Society of Nuclear Cardiology.

Heart rate increased following the injection of regadenoson. The mean maximum change in heart rate from baseline was 20 and 24 beats/min with regadenoson 400 and $500 \mu \mathrm{g}$, respectively (no significant between-dose difference). Mean systolic blood pressure (SBP) and diastolic blood pressure (DBP) decreased. The mean maximum reduction in SBP/DBP was $11 / 11 \mathrm{mmHg}$ with the $400-\mu \mathrm{g}$ dose and $17 / 9 \mathrm{mmHg}$ with the $500-\mu \mathrm{g}$ dose. Overall, patients receiving regadenoson $500 \mu \mathrm{g}$ had a greater and more sustained mean SBP reduction than those receiving regadenoson $400 \mu \mathrm{g}$. Hypotension ( $\mathrm{SBP}<90 \mathrm{mmHg}$ ) was recorded as an adverse event in one patient (in the regadenoson $500-\mu \mathrm{g}$ group). The most common side-effects reported following regadenoson administration (both doses) were chest pain (33\%), flushing (31\%), dyspnea (31\%), headache (25\%), and dizziness (19\%). The incidences of flushing, dyspnea, and dizziness were higher at the $500-\mu \mathrm{g}$ dose level. Side-effects were generally mild and transient, and there were no cases of atrioventricular block. The 400- $\mu \mathrm{g}$ dose was taken forward for evaluation in Phase III trials, due to its better tolerability over the $500-\mu \mathrm{g}$ dose.

\section{Pivotal Phase III clinical trials}

The ADenosine Versus regAdenosoN Comparative Evaluation for Myocardial Perfusion Imaging (ADVANCE MPI) 1 and
2 trials were two multicenter, international, double-blind, randomized, Phase III studies conducted in patients undergoing pharmacologic stress SPECT MPI for the evaluation of CAD (ADVANCE MPI 1, $\mathrm{n}=1231$; ADVANCE MPI 2, $\mathrm{n}=784){ }^{40,41}$ Patients had an initial MPI study with adenosine (6-minute infusion of $0.14 \mathrm{mg} / \mathrm{kg} / \mathrm{min}$, without exercise) and were randomized 2:1 to regadenoson (intravenous bolus of $400 \mu \mathrm{g}$ injected over 10 seconds; $\mathrm{n}=1337$ ) or adenosine $(n=678)$ for a second stress scan using the same radionuclide (Tc-99m sestamibi or Tc-99m tetrofosmin) and imaging protocol. Scans were evaluated visually by three expert readers blinded to treatment assignment. A 17-segment model was used for assessment, in which each segment was rated on the rest and stress images using a 5-point semi-quantitative scale for radiotracer uptake (from 0 to 4 , where $0=$ normal and 4 = absent uptake). The aim of the two studies was to demonstrate that the strength of agreement between serial initial adenosine-regadenoson images was not inferior to the strength of agreement between two serial adenosine images. The primary measure of agreement was based on the number of segments with reversible perfusion defects $(0-1,2-4$, or $\geq 5)$. The strength of agreement between adenosine-regadenoson and adenosine-adenosine images was also assessed by comparing the presence/absence of reversible defects and summed stress scores (sum of the 
segmental scores during stress, which provides an overall indication of the degree of myocardium that is compromised by infarct or ischemia), and through side-by-side analyses of the extent of ischemia.

In an analysis of pooled data from ADVANCE MPI 1 and $2(n=2015)$, mean \pm SE agreements rates for ischemia detection between sequential adenosine-adenosine and adenosine-regadenoson images were $62 \% \pm 3 \%$ and $63 \% \pm 2 \%$, respectively (difference, $0 \%$ [95\% confidence interval: $-6.2 \%, 6.8 \%$ ) (Table 1). ${ }^{41}$ The lower limit of the $95 \%$ confidence interval for the difference was above the prespecified noninferiority margin of $-13.33 \%$, indicating that regadenoson was comparable to adenosine for detecting reversible perfusion defects. Agreement rates for the presence ( $\geq 2$ segments with reversible defects) or absence (0-1 segments with reversible defects) of ischemia on sequential adenosine-adenosine and adenosine-regadenoson images were also similar (Figure 2). In side-by-side (samescreen) comparisons of sequential images, the extent of ischemia was rated as 'the same' in the two images in $82 \%$ of adenosine-adenosine and $81 \%$ of adenosine-regadenoson scans; remaining images were approximately equally distributed between the categories of 'more' ( $9 \%$ and 10\%, respectively) and 'less' ( $8 \%$ and $9 \%$, respectively) ischemia. Further evidence supporting the comparability of adenosine and regadenoson was provided by the summed stress scores, which showed good correlation between sequential studies in both randomized groups. Agreement rates between initial adenosine and randomized adenosine or regadenoson scans were comparable for subgroups defined according to age ( $<65$ and $\geq 65$ years), gender, body mass index ( $\leq 30$ and $>30 \mathrm{~kg} / \mathrm{m}^{2}$ ), and presence/absence of diabetes (Table 2). ${ }^{41}$
Thus, the ADVANCE MPI studies demonstrated that regadenoson is noninferior to adenosine in assessing the presence and extent of reversible myocardial perfusion defects in patients undergoing pharmacologic stress SPECT MPI for the evaluation of CAD. Adenosine-regadenoson agreement also appeared to be consistent across the various subgroups evaluated.

\section{Hemodynamic effects}

Time courses of the hemodynamic effects of adenosine and regadenoson in the two ADVANCE MPI studies are shown in Figure 3. The maximum mean $\pm \mathrm{SD}$ increase in heart rate was $23 \pm 11.1$ beats/min with regadenoson and $19 \pm 10.6$ beats/min with adenosine. Mean heart rate returned to within 10 beats/min of baseline by 10 minutes for regadenoson patients and by 8 minutes for adenosine patients. Thus, the increase in heart rate with regadenoson was slightly greater and more prolonged than with adenosine.

Similar reductions in SBP and DBP were observed following the administration of either regadenoson or adenosine (Figure 3). Regadenoson produced a maximum mean $\pm \mathrm{SD}$ decrease of $14 \pm 13.4 \mathrm{mmHg}$ in SBP and of $10 \pm 7.8 \mathrm{mmHg}$ in DBP; adenosine produced maximum mean reductions of $15 \pm 13.3 \mathrm{mmHg}$ in SBP and $11 \pm 8.4 \mathrm{mmHg}$ in DBP.

\section{Atrioventricular block}

Even selective $A_{2 A}$ receptor agonists may have depressive effects on the sinoatrial and atrioventricular nodes. Therefore, regadenoson is contraindicated in second- or third-degree atrioventricular block or sinus node dysfunction unless the patient has a functioning artificial pacemaker. ${ }^{24}$ In the ADVANCE MPI studies, significantly more cases of first- and second-degree atrioventricular block were noted

Table I Agreement between initial adenosine and randomized (adenosine or regadenoson) studies according to ischemia size category in a pooled analysis of ADVANCE MPI I and 2

\begin{tabular}{|c|c|c|c|c|c|c|c|c|c|}
\hline & & \multicolumn{8}{|c|}{ Randomized scan } \\
\hline & & & & & & & Reg & on & \\
\hline \multicolumn{2}{|c|}{ Ischemia extent } & No to minimal & $\begin{array}{l}\text { Small to } \\
\text { moderate }\end{array}$ & Large & $\begin{array}{c}\text { Agreement } \\
\text { rate } \pm S E\end{array}$ & No to minimal & $\begin{array}{l}\text { Small to } \\
\text { moderate }\end{array}$ & Large & $\begin{array}{l}\text { Agreement } \\
\text { rate } \pm S E\end{array}$ \\
\hline \multirow{3}{*}{ 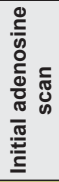 } & No to minimal & 362 & 54 & 14 & $0.84 \pm 0.02$ & 706 & 109 & 19 & $0.85 \pm 0.01$ \\
\hline & Small to moderate & 46 & 78 & 23 & $0.53 \pm 0.04$ & 101 & 144 & 49 & $0.49 \pm 0.03$ \\
\hline & Large & 11 & 16 & 27 & $0.50 \pm 0.07$ & 13 & 38 & 61 & $0.54 \pm 0.05$ \\
\hline \multicolumn{2}{|c|}{ Average agreement $\pm \mathrm{SE}$} & \multicolumn{4}{|c|}{ Adenosine $0.62 \pm 0.03$} & \multicolumn{4}{|c|}{ Regadenoson $0.63 \pm 0.02$} \\
\hline & & \multicolumn{8}{|c|}{ Agreement rate difference: $0 \%$ ( $95 \%$ confidence interval: $-6.2 .6 .8 \%)$} \\
\hline
\end{tabular}

Reproduced with permission from Cerqueira MD, Nguyen P, Staehr P, Underwood SR, Iskandrian AE, on behalf of the ADVANCE-MPI Trial Investigators. Effects of age, gender, obesity, and diabetes on the efficacy and safety of the selective $\mathrm{A}_{2 \mathrm{~A}}$ agonist regadenoson versus adenosine in myocardial perfusion imaging.J Am Coll Cardiol Img. 2008; I:307-3 I6. ${ }^{41}$ Copyright (C) 2008 American College of Cardiology Foundation. 


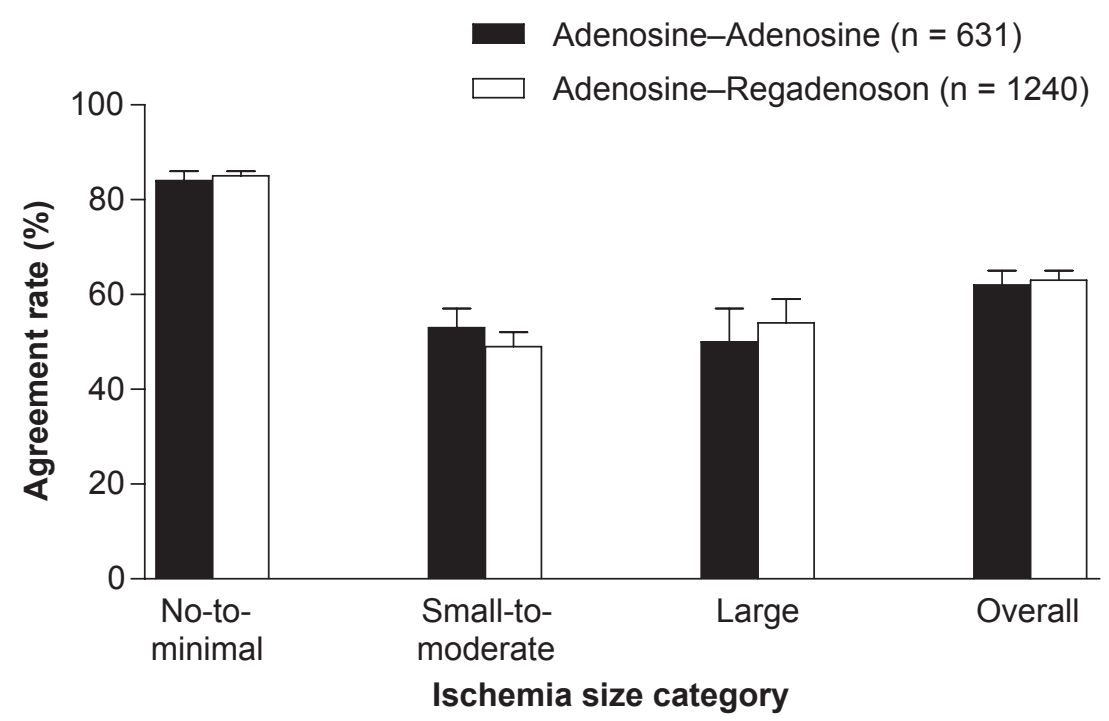

Figure 2 Agreement of adenosine (\#I) -adenosine (\#2) and adenosine (\#I)-regadenoson studies with respect to the extent of ischemia from a combined analysis of the ADVANCE MPI I and 2 trials. ${ }^{41}$ Three blinded experts independently scored images on a 5 -point scale using a 17 segment model. Ischemia was categorized as no-to-minimal (0-I segments), small-to-moderate ( $2-4$ segments), or large ( $\geq 5$ segments). Data are median $\pm S E$.

on electrocardiograms of patients with adenosine $(7.0 \%$ $[\mathrm{n}=43]$ and $1.5 \%[\mathrm{n}=9]$, respectively) than regadenoson $\left(2.8 \%[n=34]\right.$ and $0.1 \%[n=1]$, respectively) $(\mathrm{p}=0.001) .{ }^{41}$ The single regadenoson patient who had second-degree atrioventricular block with a single dropped beat at 3.5 minutes post-dose had electrocardiographic evidence of first-degree atrioventricular block at baseline. No cases of third-degree atrioventricular block were reported with either agent, although the selection of patients who did not develop second- or third-degree atrioventricular block during the first adenosine study likely impacted these results.

\section{Bronchoconstriction}

As with adenosine and dipyridamole, $\mathrm{A}_{2 \mathrm{~A}}$ receptor agonists carry a risk of bronchospasm and respiratory compromise. However, since the bronchoconstrictor effects of adenosine are thought to be mediated through $\mathrm{A}_{2 \mathrm{~B}}$ and/or $\mathrm{A}_{3}$ receptors, the risk of bronchoconstriction with agonists targeting only the $\mathrm{A}_{2 \mathrm{~A}}$ receptor may be reduced. Bronchospasm occurred in two $(<1 \%)$ adenosine patients but not in any regadenoson patients in the ADVANCE MPI trials. However, it should be noted that these trials excluded patients with known or suspected bronchoconstrictive or bronchospastic lung disease. As precautions, patients with COPD or asthma must discuss their respiratory history and the administration of pre- and post-study bronchodilator therapy with their clinician before a regadenoson MPI stress test is scheduled. ${ }^{24}$ In addition, appropriate bronchodilator therapy and resuscitative measures should be available prior to regadenoson administration to patients with known or suspected bronchoconstrictive disease, COPD, or asthma. ${ }^{24}$

\section{Adverse events}

Regadenoson has been generally safe and well tolerated in clinical trials, with no deaths attributable to the drug reported to date. ${ }^{36-45}$ Adverse events tended to be mild in intensity and most resolved spontaneously within approximately 15 minutes, except headache, which usually resolved within 30 minutes. ${ }^{24}$ Side-effects occurring at a frequency incidence of $\geq 5 \%$ with either regadenoson or adenosine in a combined analysis of the two ADVANCE MPI studies $(n=2015)$ are shown in Table $3 .{ }^{41}$ The incidences of flushing, chest discomfort, angina pectoris, chest pain, feeling hot, ST segment depression, and dysgeusia were lower with regadenoson than adenosine, whereas the incidences of dyspnea, headache, dizziness, and abdominal discomfort were higher. Although headache was also more prolonged with regadenoson than adenosine, most cases were mild and did not require treatment.

Severe and/or persistent adverse reactions to regadenoson can be reversed with aminophylline at doses of 50-250 mg, given as a slow intravenous injection. ${ }^{24,36}$ Aminophylline was administered for this purpose to $3 \%(n=46)$ of regadenoson and $2 \%(n=12)$ of adenosine patients in the ADVANCE MPI Phase III studies.

\section{Patient acceptability}

Questionnaires completed by participants in the ADVANCE MPI studies revealed that regadenoson was more acceptable 
Table 2 Ischemia size category agreement rates between initial adenosine and repeat adenosine or regadenoson images by age, gender, body mass index, and diabetes mellitus in the ADVANCE MPI I and 2 studies

\begin{tabular}{|c|c|c|c|c|c|c|c|c|c|}
\hline \multicolumn{10}{|c|}{ Agreement rate for reversible ischemia (n) } \\
\hline \multirow[b]{2}{*}{ Subgroup } & \multicolumn{4}{|c|}{ Adenosine-adenosine } & \multicolumn{4}{|c|}{ Adenosine-regadenoson } & \multirow{2}{*}{$95 \% \mathrm{Cl}$ for difference* } \\
\hline & Average & $0-1$ & $2-4$ & $\geq \mathbf{5}$ & Average & $0-1$ & $2-4$ & $\geq 5$ & \\
\hline \multicolumn{10}{|l|}{ Age, y } \\
\hline$<65$ & $56 \%(298)$ & $81 \%$ & $52 \%$ & $35 \%$ & $63 \%(544)$ & $86 \%$ & $50 \%$ & $52 \%$ & $-3 \%, 16 \%$ \\
\hline$\geq 65$ & $68 \%(333)$ & $87 \%$ & $54 \%$ & $64 \%$ & $63 \%(696)$ & $84 \%$ & $48 \%$ & $58 \%$ & $-14 \%, 4 \%$ \\
\hline \multicolumn{10}{|l|}{ Gender } \\
\hline Female & $57 \%(20 I)$ & $88 \%$ & $41 \%$ & $42 \%$ & $54 \%(376)$ & $87 \%$ & $35 \%$ & $40 \%$ & $-16 \%, 11 \%$ \\
\hline Male & $64 \%(430)$ & $82 \%$ & $57 \%$ & $52 \%$ & $65 \%(864)$ & $83 \%$ & $52 \%$ & $59 \%$ & $-6 \%, 8 \%$ \\
\hline \multicolumn{10}{|l|}{$\mathrm{BMI}, \mathrm{kg} / \mathrm{m}^{2}$} \\
\hline$\leq 30$ & $64 \%(389)$ & $86 \%$ & $59 \%$ & $47 \%$ & $62 \%(770)$ & $83 \%$ & $47 \%$ & $56 \%$ & $-10 \%, 7 \%$ \\
\hline$>30$ & $60 \%(242)$ & $82 \%$ & $44 \%$ & $55 \%$ & $64 \%(470)$ & $87 \%$ & $52 \%$ & $53 \%$ & $-7 \%, 14 \%$ \\
\hline \multicolumn{10}{|c|}{ History of diabetes } \\
\hline Yes & $59 \%(2 \mid 3)$ & $84 \%$ & $54 \%$ & $38 \%$ & $62 \%(394)$ & $81 \%$ & $48 \%$ & $56 \%$ & $-9 \%, 15 \%$ \\
\hline No & $63 \%(4 \mid 8)$ & $84 \%$ & $52 \%$ & $54 \%$ & $63 \%(846)$ & $86 \%$ & $50 \%$ & $53 \%$ & $-8 \%, 8 \%$ \\
\hline
\end{tabular}

BMI, body mass index; $\mathrm{Cl}$, confidence interval. $* 95 \% \mathrm{Cl}$ for adenosine-regadenoson average agreement rate minus repeat adenosine average agreement rate. Reproduced with permission from Cerqueira MD, Nguyen P, Staehr P, Underwood SR, Iskandrian AE, on behalf of the ADVANCE-MPI Trial Investigators. Effects of age, gender, obesity, and diabetes on the efficacy and safety of the selective $A_{2 A}$ agonist regadenoson versus adenosine in myocardial perfusion imaging. JAm Coll Cardiol Img. 2008; I:307-3I6. ${ }^{4}$ Copyright (C) 2008 American College of Cardiology Foundation.

to patients than adenosine. ${ }^{41}$ When asked how the second (adenosine or regadenoson) test compared to the initial adenosine test on a scale of 1 to 5 (where $1=$ much better and $5=$ much worse), scores were significantly lower for regadenoson than adenosine (mean $\pm \mathrm{SE}, 2.2 \pm 0.03$ versus $2.6 \pm 0.04 ; p<0.001)$. In addition, $62 \%$ of patients rated the regadenoson study as 'much better' or 'somewhat better'. Interestingly, $43 \%$ of patients rated the second adenosine study as 'much better' or 'somewhat better', possibly due to greater patient awareness of what to expect from the test. Patients were also asked to rate how they felt during the test on a 4-point scale (from $1=$ comfortable to $4=$ extremely uncomfortable); responses revealed a slightly greater level of comfort with regadenoson than adenosine (mean $\pm \mathrm{SE}$ scores, $1.7 \pm 0.02$ versus $1.9 \pm 0.03 ; p<0.001)$.

\section{Regadenoson in patients with COPD or asthma}

Two randomized, double-blind, placebo-controlled, crossover, pilot studies have been conducted to examine the safety of regadenoson in patients with reactive airways disease. ${ }^{42,43}$

One study included patients with clinically stable moderate $\operatorname{COPD}(\mathrm{n}=38)$ or severe $\operatorname{COPD}(\mathrm{n}=11)$ who were treated with a single intravenous dose of regadenoson $400 \mu \mathrm{g}$ or placebo. ${ }^{43}$ Assessment of a range of pulmonary function parameters (including forced expiratory volume in 1 second $\left[\mathrm{FEV}_{1}\right]$, forced vital capacity, respiratory rate, pulmonary examinations, oxygen saturation, and the incidence of new-onset wheezing) revealed no differences between regadenoson and placebo post-dose. There was also no significant between-treatment difference in the incidence of bronchoconstrictive reactions (defined as a decrease in $\mathrm{FEV}_{1}$ of $>15 \%$ ): 6/49 (12.2\%) following regadenoson versus $3 / 49$ (6.1\%) following placebo.

A second study involved a group of susceptible patients with mild or moderate asthma who demonstrated bronchial hyper-reactivity in a standard adenosine monophosphate challenge test $(n=48) \cdot{ }^{42}$ Each patient received a single intravenous bolus of regadenoson $400 \mu \mathrm{g}$ or placebo. Both treatments were associated with a low incidence of bronchoconstrictive reactions (a decrease of $>15 \%$ in $\mathrm{FEV}_{1}$ ): none of the patients with mild asthma and four of those with moderate asthma (2/47 [4.3\%] after regadenoson and 2/48 [4.2\%] after placebo) experienced bronchoconstriction. In addition, no significant differences between regadenoson and placebo were observed in mean $\mathrm{FEV}_{1}$ post-dosing.

In both the COPD and asthma studies, regadenoson was associated with a modest, but temporary, increase in heart rate and a higher incidence of adverse events compared with placebo. Taken together, these preliminary results suggest that the pulmonary tolerability of regadenoson in patients with mild or moderate asthma or moderate or severe COPD is good. However, since neither of these studies included imaging procedures and both included only small numbers of patients, further trials are needed to evaluate the diagnostic utility of regadenoson in patients at risk of bronchospasm. 

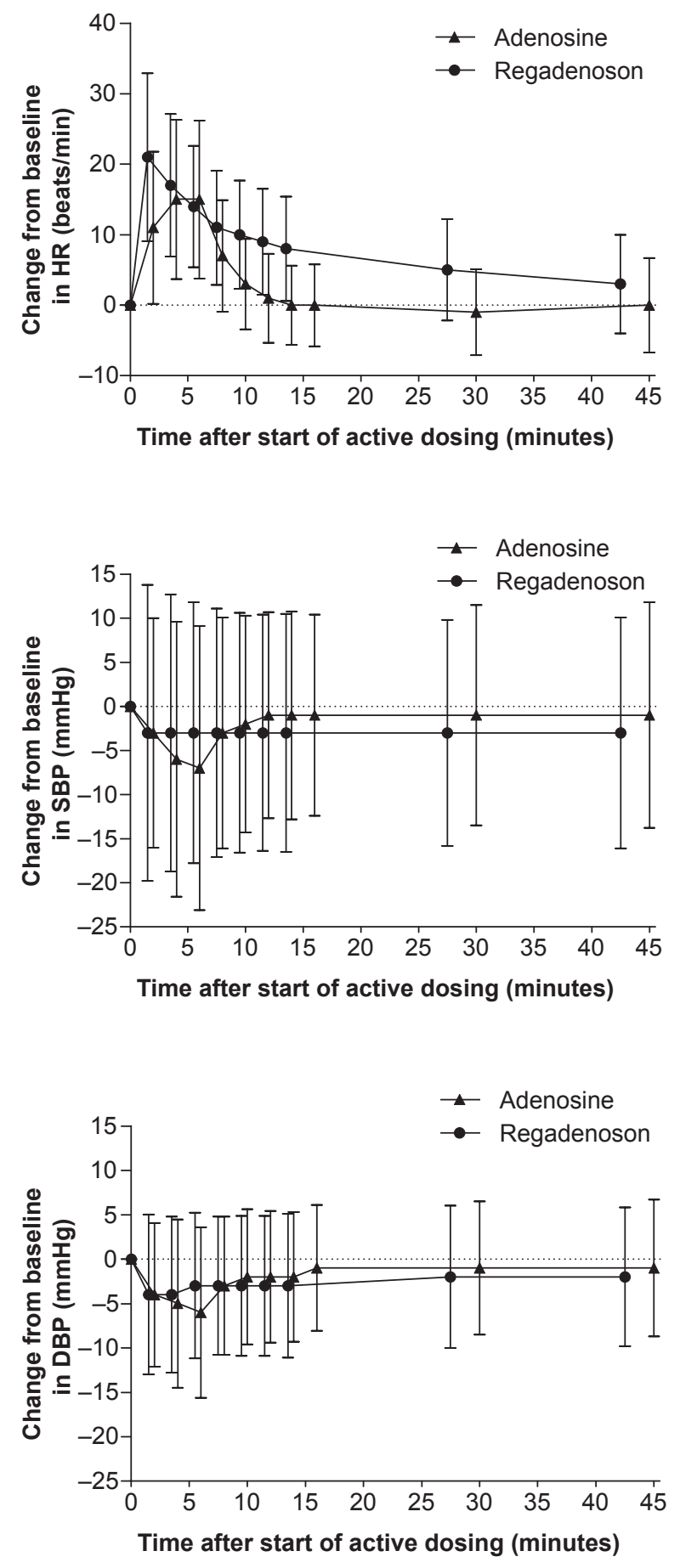

Figure 3 Time course of changes from baseline in heart rate (HR), systolic blood pressure (SBP) and diastolic blood pressure (DBP) from a combined analysis of the ADVANCE MPI I and 2 trials (unpublished). Data are mean \pm SD.

\section{Regadenoson in combination with low-level exercise}

Low-level exercise during adenosine stress MPI reduces the incidence of side-effects, attenuates the adenosine-induced fall in blood pressure, and improves image quality by decreasing hepatic and gut radiotracer uptake. ${ }^{46}$ An exploratory study has been conducted to evaluate whether similar effects are observed when regadenoson stress MPI is combined with low-level exercise. ${ }^{44}$

Patients referred for pharmacologic stress MPI underwent a standard adenosine supine stress test and were randomized (2:1) in a double-blind fashion to a second test with regadenoson $(n=39)$ or placebo $(n=21)$ combined with submaximal exercise. The second stress protocol comprised 4 minutes of slow walking on a treadmill, with intravenous injection of regadenoson $400 \mu \mathrm{g}$ or placebo at 1.5 minutes and radiotracer or placebo tracer at 2 minutes after the start of exercise.

The peak increase in heart rate from baseline was significantly greater with regadenoson-exercise (mean $\pm \mathrm{SE}$, $41.9 \pm 2.7$ beats $/ \mathrm{min})$ than adenosine alone $(21.0 \pm 2.5$ beats/ min; $p<0.001)$ or placebo-exercise $(28.9 \pm 3.7$ beats $/ \mathrm{min}$; $\mathrm{p}=0.006$ ). Similar transient SBP increases were observed during the regadenoson-exercise and placebo-exercise protocols. Symptomatic hypotension occurred in one patient with adenosine, but in none of the patients undergoing regadenoson-exercise testing.

Regadenoson-exercise testing was more tolerable and acceptable to patients than standard adenosine stress testing. Adverse events were reported by $95 \%$ of patients during the adenosine protocol, $77 \%$ during the regadenoson-exercise protocol, and 33\% during the placebo-exercise protocol. Dyspnea was the only adverse event that was more common during regadenoson-exercise testing than during the initial adenosine scan ( $54 \%$ versus $41 \%$; nonsignificant). No patient tested utilizing the regadenoson-exercise protocol experienced second-degree atrioventricular block (versus one patient with adenosine) or had a severe adverse event (versus 4 [6.7\%] patients with adenosine). In addition, $70 \%$ of patients rated the regadenoson-exercise test as 'much better' or 'somewhat better' than the adenosine test.

In side-by-side comparisons, images obtained with regadenoson-exercise MPI appeared to be of higher quality than those obtained with adenosine MPI. This was suggested by improved target-to-background radiotracer uptake ratios, better perceived overall image quality, and better perceived image quality with respect to subdiaphragmatic interference as assessed by independent readers. The sensitivity for detecting reversible perfusion defects with regadenoson-exercise was similar to that with the adenosine protocol.

The potential advantages of a stress MPI protocol combining regadenoson and low-level exercise, in terms of reduced side-effects and improved image quality, require verification in additional studies. 
Table 3 Adverse events occurring at a frequency of $\geq 5 \%$ with either regadenoson or adenosine in a pooled analysis of ADVANCE MPI I and $2^{24}$

\begin{tabular}{lll}
\hline & \multicolumn{2}{l}{ Incidence, $\mathbf{n}(\%)$} \\
\cline { 2 - 3 } Adverse event & $\begin{array}{l}\text { Regadenoson } \\
\mathbf{( n = 1 3 3 7 )}\end{array}$ & $\begin{array}{l}\text { Adenosine } \\
\mathbf{( n = 6 7 8 )}\end{array}$ \\
\hline Dyspnea & $369(28)$ & $173(26)$ \\
Headache & $342(26)$ & $113(17)$ \\
Flushing & $215(16)$ & $167(25)$ \\
Chest discomfort & $168(13)$ & $119(18)$ \\
Angina pectoris & $130(10)$ & $99(15)$ \\
Dizziness & $106(8)$ & $46(7)$ \\
Chest pain & $96(7)$ & $69(10)$ \\
Nausea & $86(6)$ & $42(6)$ \\
Feeling hot & $70(5)$ & $54(8)$ \\
ST segment depression & $67(5)$ & $45(7)$ \\
Dysgeusia & $71(5)$ & $45(7)$ \\
Abdominal discomfort & $70(5)$ & $14(2)$ \\
\hline
\end{tabular}

\section{Interaction with caffeine}

Caffeine is a nonselective, nonspecific and competitive inhibitor of all four types of adenosine receptors. As a result, it may blunt the myocardial hyperemic response to pharmacologic stress agents that exert their effects through these receptors and produce false-negative results. Ingestion of caffeine (or other methylxanthines) within 12 hours of pharmacologic vasodilator perfusion imaging is considered a contraindication to the test, ${ }^{2}$ and patients who inadvertently consume caffeinated products before the scheduled study either have their test cancelled or receive dobutamine as an alternative.

It has been shown that the consumption of one cup of coffee 1 hour prior to adenosine SPECT perfusion imaging does not affect the extent or severity of reversible ischemic defects. ${ }^{47}$ A recent double-blind, randomized, placebo-controlled, crossover study has also provided some indication that prior caffeine intake may not limit the clinical use of regadenoson. ${ }^{45}$

Healthy regular coffee drinkers $(n=41)$ received either a caffeine capsule (200 mg po; equivalent to two cups of coffee) or placebo 2 hours before regadenoson administration (400 $\mu \mathrm{g}$ intravenously over 10 seconds). Myocardial blood flow was measured at rest and immediately after the regadenoson injection using positron emission tomography with radiolabeled water. The effect of regadenoson on coronary flow reserve (myocardial blood flow stress-to-rest ratio) was similar with and without caffeine (mean \pm SE, $2.75 \pm$ 0.16 versus $2.97 \pm 0.16$; nonsignificant).

Caffeine blunted the increase in heart rate induced by regadenoson by 20 beats/min ( $<<0.001$ ), but had no effect on SBP or DBP. Overall adverse event rates were similar in the caffeine and placebo groups, with dyspnea (56\%), palpitations (49\%), flushing (30\%), headache

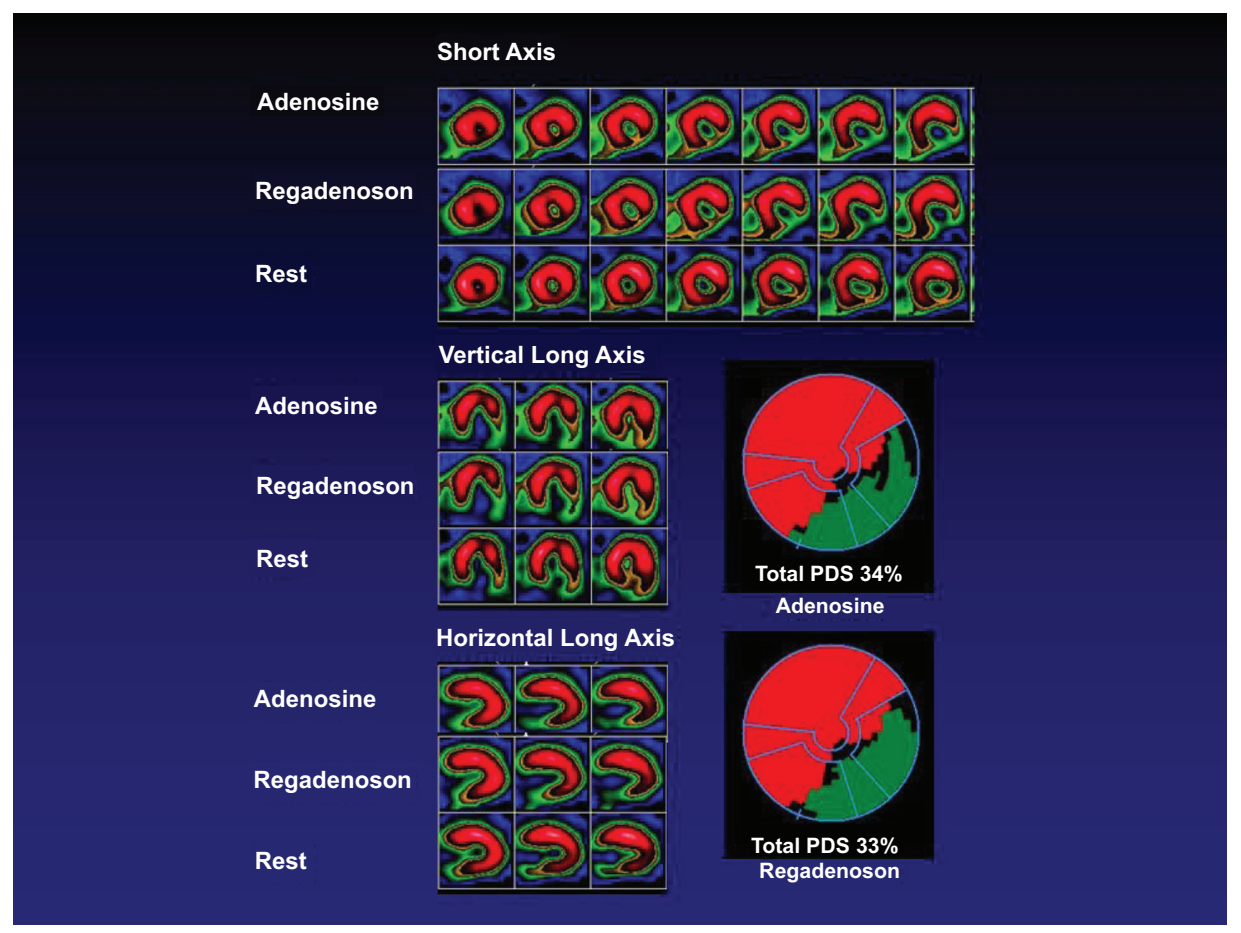

Figure 4 Stress images after either an adenosine (top row) or regadenoson (middle row) stress demonstrating a partially reversible defect in the inferior and inferolateral walls. The defect size, severity, and location are virtually identical with each method of stress imaging, as confirmed on the polar plot. Figure courtesy of JJ Mahmarian (with permission). 
(28\%), sensation of heaviness (28\%), and paresthesia (19\%) being the most commonly reported adverse events. However, caffeine ingestion alleviated the severity of side-effects (notably headache; $p=0.012$ ) and improved tolerability (as assessed by patient questionnaires; $\mathrm{p}<0.001)$.

These results suggest that moderate caffeine consumption does not significantly affect regadenoson-induced coronary vasodilation in healthy individuals and may also improve the tolerability of regadenoson stress testing. Whether caffeine affects the diagnostic accuracy of detecting reversible ischemic defects in patients with CAD using regadenoson stress MPI merits further investigation.

\section{Conclusion}

Regadenoson is the first selective $\mathrm{A}_{2 \mathrm{~A}}$ receptor agonist to be approved for clinical use as a pharmacologic stress agent for the detection of ischemia in patients with limited exercise capacity using MPI. It is administered at a standard, fixed dose $(400-\mu \mathrm{g}$ in $5 \mathrm{~mL}$ ) by an intravenous bolus over 10 seconds. ${ }^{24}$ Dose adjustment by body weight or renal function is not necessary. Regadenoson, therefore, simplifies drug delivery, decreases the risk of dose-calculation errors, and does not require specific infusion equipment (such as specialized tubing and computerized pumps).

In the pivotal Phase III ADVANCE MPI 1 and 2 studies, ${ }^{40,41}$ images acquired during regadenoson stress SPECT MPI were reproducible and comparable to those acquired with adenosine for evaluating the extent of coronary perfusion defects in patients with suspected CAD. ${ }^{39-41}$ Hemodynamic effects of regadenoson included an increase in heart rate and a slight decrease in blood pressure. Adverse events were mainly mild, transient, and self-limiting. Sideeffects associated with peripheral vasodilation and activation of other adenosine receptor subtypes (eg, chest pain, dyspnea, and flushing) occurred less frequently with regadenoson than adenosine, although headache and gastrointestinal discomfort were more common. There were no cases of bronchoconstriction and a single case of second-degree atrioventricular block.

Therefore, regadenoson, a low-affinity, potent, selective $\mathrm{A}_{2 \mathrm{~A}}$ adenosine receptor agonist, represents an alternative pharmacologic stress agent for use in conjunction with SPECT MPI. The standard dose of $400 \mu \mathrm{g}$ delivered as an intravenous bolus over 10 seconds, provides good quality images yielding accurate diagnostic information, is safe, well tolerated, and easy to administer. Studies are required to further evaluate the potential use of regadenoson as a stress agent in patients with bronchospastic lung disease or atrioventricular block, in combination with submaximal exercise, and following caffeine consumption. The impact of regadenoson-induced tachycardia on its clinical utility in patients with left bundle branch block also requires additional investigation.

\section{Acknowledgment}

Editorial support was provided by Elaine F Griffin, MA, DPhil, a medical writer at Envision Pharma.

\section{Disclosures}

The author currently serves on the Regadenoson Advisory Board for Astellas Pharma US, Inc and is part of the speakers' bureau. Previously, Dr. Hendel was an investigator and steering committee member for CV Therapeutics, the developers of CVT-7146 (regadenoson). He also is part of advisory groups for GE Healthcare and PGx Health.

\section{References}

1. Klocke FJ, Baird MG, Bateman TM, et al. ACC/AHA/ASNC guidelines for the clinical use of cardiac radionuclide imaging. A report of the American College of Cardiology/American Heart Association Task Force on Practice Guidelines (ACC/AHA/ASNC Committee to Revise the 1995 Guidelines for the Clinical Use of Cardiac Radionuclide Imaging). American College of Cardiology Web Site. Available at: http://www.acc.org/qualityandscience/clinical/guidelines/radio/index. pdf. Accessed 3 June, 2008.

2. DePuey EG, Corbett JR, Friedman JD, et al. Imaging guidelines for nuclear cardiology procedures. A report of the American Society of Nuclear Cardiology Quality Assurance Committee. American Society of Nuclear Cardiology. Available at: http://www.asnc.org/ imageuploads/ImagingGuidelinesComplete0624081.pdf. Accessed 3 November, 2008.

3. Klocke FJ, Baird MG, Lorell BH, et al. ACC/AHA/ASNC guidelines for the clinical use of cardiac radionuclide imaging-executive summary: a report of the American College of Cardiology/American Heart Association Task Force on Practice Guidelines (ACC/AHA/ASNC Committee to Revise the 1995 Guidelines for the Clinical Use of Cardiac Radionuclide Imaging). J Am Coll Cardiol. 2003;42:1318-1333.

4. Hendel RC. Utilization management of cardiovascular imaging: pre-certification and appropriateness. J Am Coll Cardiol Img. 2008; $1: 241-248$.

5. Brindis RG, Douglas PS, Hendel RC, et al. ACCF/ASNC appropriateness criteria for single-photon emission computed tomography myocardial perfusion imaging (SPECT MPI): a report of the American College of Cardiology Foundation Quality Strategic Directions Committee Appropriateness Criteria Working Group and the American Society of Nuclear Cardiology endorsed by the American Heart Association. J Am Coll Cardiol. 2005;46:1587-1605.

6. Cerqueira MD, Verani MS, Schwaiger M, Heo J, Iskandrian AS. Safety profile of adenosine stress perfusion imaging: results from the Adenoscan Multicenter Trial Registry. J Am Coll Cardiol. 1994; 23:384-389.

7. Ranhosky A, Kempthorne-Rawson J, and the Intravenous Dipyridamole Thallium Imaging Study Group. The safety of intravenous dipyridamole thallium myocardial perfusion imaging. Circulation. 1990;81:1205-1209. 
8. Astellas Pharma US Inc. Adenoscan ${ }^{\circledR}$ (adenosine injection) United States prescribing information. July 2005. Available at http://www. astellas.us/docs/adenoscan.pdf. Accessed 7 July, 2008.

9. Bedford Laboratories ${ }^{\mathrm{TM}}$. Dipyridamole injection. For intravenous injection. United States prescribing information. February 2006. Available at http://www.bedfordlabs.com/products/inserts/Div-DPP05. pdf. Accessed 11 August, 2008.

10. Elhendy A, Valkema R, van Domburg RT, et al. Safety of dobutamineatropine stress myocardial perfusion scintigraphy. $\mathrm{J} \mathrm{Nucl} \mathrm{Med}$. 1998;39:1662-1666.

11. Elhendy A, Bax JJ, Poldermans D. Dobutamine stress myocardial perfusion imaging in coronary artery disease. $J$ Nucl Med. 2002;43:1634-1646.

12. Bedford Laboratories ${ }^{\mathrm{TM}}$. DOBUTamine injection USP. United States prescribing information. June 2005. Available at http://www.bedfordlabs. com/products/inserts/Div-DBP04.pdf. Accessed 11 August, 2008.

13. Belardinelli L, Shryock JC, Snowdy S, et al. The $\mathrm{A}_{2 \mathrm{~A}}$ adenosine receptor mediates coronary vasodilation. J Pharmacol Exp Ther. 1998;284:1066-1073.

14. Stone TW. Receptors for adenosine and adenine nucleotides. Gen Pharmacol. 1991;22:25-31.

15. Klotz KN. Adenosine receptors and their ligands. Naunyn Schmiedebergs Arch Pharmacol. 2000;362:382-391.

16. Belardinelli L, Linden J, Berne RM. The cardiac effects of adenosine. Prog Cardiovasc Dis. 1989;32:73-97.

17. Olsson RA, Pearson JD. Cardiovascular purinoceptors. Physiol Rev 1990;70:761-845.

18. Gaspardone A, Crea F, Tomai F, et al. Muscular and cardiac adenosineinduced pain is mediated by A1 receptors. $\mathrm{J} \mathrm{Am} \mathrm{Coll} \mathrm{Cardiol}$. $1995 ; 25: 251-257$

19. Feoktistov I, Biaggioni I. Adenosine A2B receptors. Pharmacol Rev. 1997;49:381-402.

20. Baraldi PG, Cacciari B, Romagnoli R, et al. A(3) adenosine receptor ligands: history and perspectives. Med Res Rev. 2000;20:103-128.

21. Hendel RC, Jamil T, Glover DK. Pharmacologic stress testing: new methods and new agents. J Nucl Cardiol. 2003;10:197-204.

22. Cerqueira MD. The future of pharmacologic stress: selective $A_{2 A}$ adenosine receptor agonists. Am J Cardiol. 2004;94(Suppl):33D-40D.

23. Cerqueira MD. Advances in pharmacologic agents in imaging: new $\mathrm{A}_{2 \mathrm{~A}}$ receptor agonists. Curr Cardiol Rep. 2006;8:119-122.

24. Astellas Pharma US Inc. Lexiscan ${ }^{\mathrm{TM}}$ (regadenoson) injection. United States prescribing information. April 2008. Available at http://www. astellas.us/docs/lexiscan.xml. Accessed 19 August, 2008.

25. Glover DK, Ruiz M, Yang JY, et al. Pharmacological stress thallium scintigraphy with 2-cyclohexylmethylidenehydrazinoadenosine (WRC-0470). A novel, short-acting adenosine $\mathrm{A}_{2 \mathrm{~A}}$ receptor agonist. Circulation. 1996;94:1726-1732.

26. Martin PL, Barrett R, Sykes A, Droppleman DA, Wright KF, Mossem D. Pharmacology and toxicology of the $\mathrm{A}_{2 \mathrm{~A}}$-adenosine receptor agonist 2- [(cyclohexylmethylene)hydrazino]adenosine (MRE-0470) in the rat Drug Dev Res. 1997;42:76-85.

27. Martin PL, Barrett RJ, Linden J, Abraham WM. Pharmacology of 2-cyclohexylmethylidenehydrazinoadenosine (WRC-0470), a novel, short-acting adenosine $\mathrm{A}_{2 \mathrm{~A}}$ receptor agonist that produces selective coronary vasodilation. Drug Dev Res. 1997;40:313-324.

28. Barrett RJ, Lamson MJ, Johnson J, Smith WB. Pharmacokinetics and safety of binodenoson after intravenous dose escalation in healthy volunteers. J Nucl Cardiol. 2005;12:166-171.

29. Hodgson JM, Dib N, Kern MJ, Bach RG, Barrett RJ. Coronary circulation responses to binodenoson, a selective adenosine $\mathrm{A}_{2 \mathrm{~A}}$ receptor agonist. Am J Cardiol. 2007;99:1507-1512.

30. Udelson JE, Heller GV, Wackers FJ, et al. Randomized, controlled dose-ranging study of the selective adenosine $\mathrm{A}_{2 \mathrm{~A}}$ receptor agonist binodenoson for pharmacological stress as an adjunct to myocardial perfusion imaging. Circulation. 2004;109:457-464.

31. Busse W, Weiler J, Murray JJ, et al. Administration of binodenoson, a selective adenosine A2a receptor agonist pharmacologic stress agent, is safe in asthmatics. $J$ Nucl Cardiol. 2007;14:S102 [abstract 101.114].
32. Udelson JE, Iteld B, Weiland FL, et al. Efficacy and safety of the selective adenosine $\mathrm{A}_{2 \mathrm{~A}}$ receptor agonist binodenoson for pharmacologic stress: results of a Phase 3, randomized, double-blind, risk-stratified, active-controlled, crossover trial. 57th Annual Scientific Session of the American College of Cardiology, Chicago, Illinois, USA. 29 March-1 April 2008. Late-breaking abstract 409-05.

33. Glover DK, Ruiz M, Takehana K, et al. Pharmacological stress myocardial perfusion imaging with the potent and selective $\mathrm{A}(2 \mathrm{~A})$ adenosine receptor agonists ATL193 and ATL146e administered by either intravenous infusion or bolus injection. Circulation. 2001;104: 1181-1187.

34. Zablocki J, Palle V, Blackburn B, et al. 2-substituted pi system derivatives of adenosine that are coronary vasodilators acting via the $\mathrm{A}_{2 \mathrm{~A}}$ adenosine receptor. Nucleosides Nucleotides Nucleic Acids. 2001;20:343-360.

35. Gao Z, Li Z, Baker SP, et al. Novel short-acting $A_{2 A}$ adenosine receptor agonists for coronary vasodilation: inverse relationship between affinity and duration of action of $\mathrm{A}_{2 \mathrm{~A}}$ agonists. J Pharmacol Exp Ther. 2001;298:209-218.

36. Lieu HD, Shryock JC, von Mering GO, et al. Regadenoson, a selective $A_{2 \mathrm{~A}}$ adenosine receptor agonist, causes dose-dependent increases in coronary blood flow velocity in humans. J Nucl Cardiol. 2007;14:514-520.

37. Gordi T, Frohna P, Sun HL, Wolff A, Belardinelli L, Lieu H. A population pharmacokinetic/pharmacodynamic analysis of regadenoson, an adenosine $\mathrm{A}_{2 \mathrm{~A}}$-receptor agonist, in healthy male volunteers. Clin Pharmacokinet. 2006; 45:1201-1212.

38. Gordi T, Blackburn B, Lieu H. Regadenoson pharmacokinetics and tolerability in subjects with impaired renal function. J Clin Pharmacol. $2007 ; 47: 825-833$

39. Hendel RC, Bateman TM, Cerqueira MD, et al. Initial clinical experience with regadenoson, a novel selective $A_{2 \mathrm{~A}}$ agonist for pharmacologic stress single-photon emission computed tomography myocardial perfusion imaging. J Am Coll Cardiol. 2005;46:2069-2075.

40. Iskandrian AE, Bateman TM, Belardinelli L, et al. Adenosine versus regadenoson comparative evaluation in myocardial perfusion imaging: results of the ADVANCE phase 3 multicenter international trial. J Nucl Cardiol. 2007;14:645-658.

41. Cerqueira MD, Nguyen $\mathrm{P}$, Staehr P, Underwood SR, Iskandrian AE, on behalf of the ADVANCE-MPI Trial Investigators. Effects of age, gender, obesity, and diabetes on the efficacy and safety of the selective $\mathrm{A}_{2 \mathrm{~A}}$ agonist regadenoson versus adenosine in myocardial perfusion imaging. J Am Coll Cardiol Img. 2008;1:307-316.

42. Leaker BR, O'Connor B, Hansel TT, et al. Safety of regadenoson, an adenosine $\mathrm{A}_{2 \mathrm{~A}}$ receptor agonist for myocardial perfusion imaging, in mild asthma and moderate asthma patients: a randomized, double-blind, placebo-controlled trial. J Nucl Cardiol. 2008;15:329-336.

43. Thomas GS, Tammelin BR, Schiffman GL, et al. Safety of regadenoson, a selective adenosine $\mathrm{A}_{2 \mathrm{~A}}$ agonist, in patients with chronic obstructive pulmonary disease: a randomized, double-blind, placebo-controlled trial (RegCOPD trial). J Nucl Cardiol. 2008;15:319-328.

44. Thomas GS, Thompson RC, Miyamoto MI, et al. The RegEx trial: a randomized, double-blind, placebo- and active-controlled pilot study combining regadenoson, a selective $\mathrm{A}_{2 \mathrm{~A}}$ adenosine agonist, with lowlevel exercise, in patients undergoing myocardial perfusion imaging J Nucl Cardiol. 2009;16(1):63-72.

45. Gaemperli O, Schepis T, Koepfli P, et al. Interaction of caffeine with regadenoson-induced hyperemic myocardial blood flow as measured by positron emission tomography: a randomized, double-blind, placebocontrolled crossover trial. J Am Coll Cardiol. 2008;51:328-329.

46. Elliott MD, Holly TA, Leonard SM, Hendel RC. Impact of an abbreviated adenosine protocol incorporating adjunctive treadmill exercise on adverse effects and image quality in patients undergoing stress myocardial perfusion imaging. J Nucl Cardiol. 2000;7:584-589.

47. Zoghbi GJ, Htay T, Aqel R, Blackmon L, Heo J, Iskandrian AE. Effect of caffeine on ischemia detection by adenosine single-photon emission computed tomography perfusion imaging. J Am Coll Cardiol. 2006;47:2296-2302. 
